\title{
XXII.
}

(Aus dem k. u. k. Garnisons-Spitale Nr. 1 in Wien.)

\section{Die Beurtheilung ein- und beiderseitiger Tanbheit.}

\author{
Von \\ Dr. Carl Biehl, \\ Oberarzt, Vorstand der Ohrenabtheilung.
}

Ausser dem Militärarzte wird höchstens noch der Kassenarzt einer Versicherungs-Gesellschaft so häufig in die Lage kommen, die Angaben der Leute, welche, sei es dass sie irgend ein Trauma erlitten haben oder nicht, plötzlich taub geworden sein wollen, auf ihre Wabrheit zu prüfen und sodann ein oft folgenschweres Gutachten abzugeben. Dass es sowohl Pflicht als auch für den guten Namen des Arztes sehr nothwendig ist, bei solchen Untersuchungen und Gutachten äusserst genau vorzugehen, damit er nicht unrecht thut oder gar eine grobe Enttäuschung erlebt, braucht nicht erst betont zu werden.

Was nun das Trauma anbelangt, so ist es beim Militär wohl die Ohrfeige, welche in den meisten Fällen als solches zu eruiren ist. „Das Ohr ist auch leider nur zu häufig das Ziel, wohin nicht blos die leidenschaftlichen Ausbrüche des Zornes, sondern anch die mit Ueberlegung geplanten Züchtigungen ihren Angriff richten. Wir finden daher hier an der linken Seite häufiger als an der rechten die Zeichen der vorausgegangenen Insulte." "I) Die schädlichen Wirkungen dieser Insulte können sich durch directe oder indirecte Fortplanzung der Gewalt auf alle Theile sowohl des äusseren, des mittleren, als auch des inneren Ohres in mannigfacher Weise geitend machen. Den objectiven Befund zu constatiren, zumal wenn nur das äussere, in den allermeisten Fällen, wenn auch das Mittelohr getroffen ist, bietet keine grossen Schwierigkeiten. Schwieriger jedoch gestaltet sich die Diagnose schon bei jenen Verletzungen, welche auch das innere Ohr in

1) Kirchuer: Handibuch von Schwartze. Bd. II. S. 5 . Archiv f, Ohrenheilkunde. XLIII. Bd. 
Mitleidenschaft gezogen baben, und zwar besonders dann, wenn schon geraume Zeit seither verstrichen ist, und die eventuell zu constatirenden, auf eine Affection des inneren Ohres hinweisenden Symptome, wie Bewusstlosigkeit, Schwindel, Erbrechen, wieder geschwunden sind. Die zurückgebliebene ein- oder beiderseitige Schwerhörigkeit oder gar Taubheit nachzuweisen, ist schon deshalb immer schwieriger, da es ja hierfür keine objective Untersuchungsmethode giebt, und man auf die Angaben und den guten oder bösen Willen der zu Untersuchenden angewiesen ist. Dass daher oftmals sehr unvollkommene Resultate zu erzielen sind, sei es infolge einer untiberwindlichen Indolenz und Unfähigkeit, sei es aus Mangel an gutem Willen von Seiten des auf seine Angaben zu Prüfenden - letzteres wird dem Militärarzte wohl am bäufigsten vorkommen - , darf einem also nicht Wunder nehmen.

Wird einseitige Taubheit simulirt, so ist dies oft nicht schwer nachzuweisen, und sind hierfür mannigfache Verfahren angegeben. Moos ${ }^{1}$ ), Schwartze ${ }^{2}$, Goggin ${ }^{3}$ ), Miller ${ }^{4}$ ), Teuber ${ }^{5}$ ), Gellé( $)$, Gruber ${ }^{7}$ ) u. a. m. haben derartige Entlarvungsversuche bekannt gemacht. Am Moskauer Congresse ist neverdings ein "Apparat zur Untersuchung auf simulirte einseitige Taubheit" von Herrn Stabsarzt Dr. K ale i c demonstrirt worden. ${ }^{8}$ ) Es ist dies ein transportables Handtelephon, welches seinem $\mathrm{Z}$ wecke vollkommen entsprechen kann, wenn beide Untersucher gegenseitig, als auch auf den Apparat geschult sind. Hier sowohl wie bei den fruher angeführten Versuchen besteht der Witz der Entlarvang darin, dass durch gleichzeitig gesprochene Worte sowohl in das gesunde wie in das angeblich taube Ohr der Simulant derart ermüdet und schliesslich verwirrt wird, dass er beschämend den Schwindel zugestehen muss. Um dies aber auch in jedem Falle zu erreichen, mutssen beide Sprecher möglichst ähnliche Stimmen haben, gleichzeitig im Tacte und gleichstark sprechen. Aehnliche Stimmen sind deshalb erforderlich,

1) Ein einfaches Verfahren zur Diagnose einseitig simulirter Taubheit. A. f. A. u, 0. Bd. 1 .

2) Die chirurgischen Krankheiten des Ohres.

3) Eine neue Prufungsmethode auf simulirte einseitige Taubheit. Z. f. 0 . Bd. VIII.

4) Berliner klin. Wochenschrift. 1869. Nr. 15.

5) Ebenda. Nr. 9.

6) Gaz. méd. de Paris 1877. Nr. 8.

7) Zur Hörprufung. M. f. 0, 1885. Nr. 2.

8) Wiener med. Wochenschrift. Nr. 42, 1897. 
weil sich der angeblich Taube nur zu leicht den Stimmenklang: des in das angeblich taube Obr Sprechenden merken kann, und es ihm so leicht möglich ist, dieses $\mathrm{Obr}$ auszuschalten, ibn zu überhören.

Bei den unzähligen und manchmal recht mïhevollen Hörprifungen auf der hiesigen Ohrenabtheilung kam ich nun in solchen Fällen auf einen kleinen, ich glaube, Vortheil, welcher sich mir bis jetzt fast immer bewährte und nur selten im Stiche liess. Der Vortheil der Einfachheit und Billigkeit zu mindest lässt sich nicht leugnen. Aufmerksam auf diese Methode machte mich Herr Stabsarzt Dr. S pi e.gel, welcher oftmals meinen Hörprüfungen beiwohnte und manchmal mein vergebliches Bemühen, einen offenbaren Schwindler zu ertappen, mit ansah. Der Vorgang ist folgender:

Zuerst wird die Hörschärfe des gesunden Obres geprüft. Sodann steckt man, ohne dass es der Untersuchte wahrnimmt, ähnlich einem Ohrtrichter ein ca. $2 \mathrm{Cm}$. langes Stück eines Kantschukschlauches, dessen Weite entsprechen muss der des äusseren Gehörganges, möglichst tief in diesen, und zwar a uf der gesunden Seite. Der Untersucher verdeckt nun mit der einen Hand die Augen des zu Untersuchenden, mit einer Fingerkuppe der anderen Hand spielt er mit dem freien Ende des Kantschukschlauches, und zwar derart, dass während der nun vorzunehmenden Hörprïfung das Lumen desselben bald geöffnet, bald geschlossen ist. Die Flüsterzahlen oder - Worte werden von einer beliebigen anderen Person gesprochen, welche, besonders wenn es sich um angebliche Schwerbörigkeit handelt, oftmals und unmerklich!) die Entfernung vom angeblich kranken Ohre wechselt. Der Kautschukschlauch erzengt das Gefühl des vollkommenen Verschlusses ${ }^{2}$ ); spielt die Fingerkuppe geschickt, und hierzu bedarf es keiner Kunst, so ist es selbst sehr vorsichtigen Leaten unmöglich, ibre angebliche Schwerhörigkeit oder gar Taubheit beizubehalten. Die Simulation der letzteren ist erwiesen, wenn am angeblich tauben Ohre bei offenem Kautschukschlauche nichts gehört wird. Wie erwähnt, hat mich diese Art der Untersuchung noch selten im Stiche gelassen; den Vortheil der Einfachbeit hat sie sicherlich.

1) Durch Gummisohlen oder einfacher durch Galoschen ist dies leicht zu erreichen.

2) Der Kantschukschlauch muss eng passen und kann za diesem Zwecke auch eingefettet werden. 
Bedeutend schwieriger ist jedoch der Nachweis simulirter bilateraler Taubheit. Hier lässt einen oft angewandte Güte oder Strenge im Stiche und bilft nur List; doch auch diese führt häufig genug nicht zum Ziele. Zeigt der angeblich Taube einen festen Willen, so ist jedes Bemühen ausgeschlossen. In derartigen Fällen ist es dann sicherlich vortheilhafter und zugleich weniger verantwortlich, wenn der Mann vom Militärdienste zeitlich befreit wird, jedoch im Civile unter Controle bleibt. So kann es eben dann wiederum nicht vorkommen, dass Jemand als Simulant grosser Schwerhörigkeit oder Taubheit erklärt und verurtheilt wird, welcher in der That, wenn auch auf nicht allzu bäufige Weise, sein Gehör verloren hat. „Unrecht thun, thut weh", sagt ja ein altes Sprichwort.

Bekannte Thatsache ist, dass directe Schädeltraumen, selbst wenn keine Verletzungen des knöchernen Gerüstes, auch keine der umgebenden Weichtheile nachzuweisen sind, alle Grade der Gehörstörungen bis zur vollständigen Taubheit verursachen können. Für jene Fälle nun, fur welche eine Labyrintbblutung als anatomische Grundlage hierfür anzunebmen ist, hat man auch eine vollauf befriedigende Erklärung, nicht so aber für die reinen Commotionen des Labyrinthes ohne anatomische Läsion. Dass letztere, ähnlich der Commotio cerebri, vorkommen, ist nicht von der Hand zu weisen. „Die Unterscheidungsmerkmale zwischen Blutung und einer Commotion sind in vieler Hinsicht anfechtbar. Plausibel wäre die Diagnose der Blutung am ehesten dort, wo es sich nicht um eine gleichmässige Perception, sondern um beschränkte Ausfallserscheinungen handelt." Gleichgewichtsstö. rungen, besonders aber lang anhaltender Schwindel machen die Annahme einer Blutung sehr wahrscheinlich.

Während also die Labyrinthblutung nachzuweisende anatomische Läsionen hinterlässt, werden bei der Commotion „, moleculare Veränderungen der nervösen Formbestandtheile " ${ }^{2}$ ) oder ,plötzlicbe Lageveränderungen des Nervenendapparates infolge der Erschütterung der Labyrinthflussigkeit" 3) angenommen. Welche von den beiden Anschaungen Berechtigung hat, werd en künftige Untersuchungen zeigen. Bei der Labyrinthblutung ganz sicber, wahrscheinlicb auch bei der reinen Commotion sind materielle Veränderungen die Ur-

1) Brieger: Klinische Beiträge zur Obrenheilkunde, Wiesbaden.

2) Schwartze: Die chirurg. Krankheiten des Ohres.

3) Politzer: Lebrbuch. 1I. Aufl. 
sache der Gehörstörung; es mïssen aber auch als Folgezustände directer Schädeltraumen ohne nacbzuweisende grobe Verletzung rein functionelle Störungen, ;als Theilerscheinung der traumatischen Neurose, angenommen werden. Bis in die neueste Zeit findet man über diesen Gegenstand 'sowohl in den Lebrbüchern der Neuropathologie wie der Otologie entweder gar nichts oder nur ganz kurze Andeutungen. Erst Gradenigo $0^{1}$ und Brieger (l. c.) bringen ausfiuhrlichere Arbeiten bieriber; namentlich behandelt ersterer eingehend denselben, und findet man daselbst auch die ganze diesbezügliche Literatur angegeben. Er sammelte alle hierher gehörigen, bisher bekannten Beobachtungen und fand, „dass unter 73 Fällen von männlicher oder weiblicher Hysterie oder von Hystero-Traumatismus bei 32 (43,8 Proc.) Störungen der Gehörsensibilität angegeben werden."

Die auf Traumen folgenden Taubheiten, welche keine materiellen Veränderangen, Labyrinthblutung oder Commotion, als Ursache haben, kann man nach Gradenigo in 2 Abtheilungen unterbringen:

a) diejenigen, welche, wie bei Hysterie, eines der Symptome der sensitiv-sensoriellen Anästhesie ausmachen;

b) diejenigen, welche im Krankheitsbilde das hauptsächliche, bisweilen einzige Phänomen der Hysterie darstellen und an specielle Zustände des Traumas und des Obres gebunden sind.

Aus dieser Eintheilung ist zu entnehmen, dass sie die Ansicht Charcot's, welcher die tranmatische Neurose der Hysterie analog betrachtet (Hystero-Traumatismus), als Grundlage hat.

In Hinblick auf die später zu erwähnende Beobachtung kommen hier nur die Fälle zur Betrachtung, welche Taubheit als das einzige Symptom der traumatischen Neurose constatiren lassen.

Itard ${ }^{2}$ ) berichtet von einem Kranken, welcher 8 Tage nach einer leichten Verwundung am rechten Obre vollständig taub geworden.

Urbantsehits $\mathrm{ch}^{3}$ ) beobachtete beiderseitige vollständige Taubheit nach einem schwach gefübrten Schlag mit einem Löffel auf das rechte Stirnbein bei einem 9 Jahre alten Knaben.

1) Handbuch v. Schwartze. Bd. I. S. 498; Hang's klinische Vorträge. Bd. I. 13. Heft.

2) Traité 1821.

3) Dieses Archiv. Bd. XVI. S. 183. 
Politzer ${ }^{1}$ ) beobachtete ebenfalls vollständige Taubheit nach einem Stoss auf den Kopf; langsam wiederkehrendes Gehör, bis am 23. Tage dasselbe unter starkem Schwindelanfall und dem Eindrucke einer starken Erschutterung ganz wiederkehrte.

$D$ él i e ${ }^{2}$ ) erzählt von. einem Kranken, welcher nach einem Schlag auf's Hinterhaupt sofort nicht sprechen konnte und vollständig taub war (complètement sourd). Kein Kopfschmerz, kein Schwindel, kein Erbrechen und keine subjectiven Empfindungen im Ohre (la logoplégie est manifeste). Nach 8 Tagen Besserung des Geböres, jedoch nicht der Sprache; nach 2 Monaten Sturz ins Wasser, worauf Sprache und Gehör vollkommen wiederkehrten.

Hierher gehören anch noch die Fälle von $\left.\mathrm{Badel}{ }^{3}\right), \mathrm{Roosa}^{4}$ ) und Gradenigo $0^{5}$ ).

Ausser den hier angeführten Bobachtungen wird es wohl nicht so leicht möglich sein, noch eine grosse Anzahl hierhergehöriger zu finden, d. h. solcher, bei denen Taubheit sich als das einzige Symptom des Hystero. Traumatismus manifestirt. Dies sowohl wie auch der Umstand, dass über die Bedeutung des Traumas bei den traumatischen Neurosen hente noch verschiedene Ansichten herrschen, rechtfertigt die Bekanntgabe eines neuen, bierher gehörigen Falles, welcher durch 5 Monate in ununterbrochener Beobachtung stand und begutachtet werden musste. Es war ein Untersuchungshäftling, welcher bereits zweimal wegen Simulation von grosser Sehwerhörigkeit abgestraft worden war und nun abermals eben deshalb in Untersuchung stand. Aus den Acten war kurz folgendes zu entnehmen:

Dragoner S. erhielt Anfangs December 1894 eine Ohrfeige auf das linke Obr. Er machte hiervon keine Anzeige und wurde erst 8 Tage später ins zugehörige Spital abgegeben, als seine Schwerhörigkeit bei der Escadron auffiel. Im Spitale wurde eine Ruptur des linken Trommelfelles constatirt, nnd ein Causalnexus zwischen dieser und der Ohrfeige zugegeben. "Die Hörprüfung ergab eine Herabsetzung der Hörschärfe, welche jedoch wegen der stark subjectiven Färbung der Angaben nicht genau controlirbar ist." $\mathrm{S}$. wurde gegen Ende Januar, ,vollkommen geheilt" zur Truppe geschickt, jedoch ganz kurze Zeit hernach, Mitte Februar, wegen ,angeblicher Schwerhörigkeit" wiederum dem Spitale übergeben. So wechselte sein Aufenthalt zwischen Spital und Escadron, bis er endlich, wegen hartnäckiger Simulation von Taubheit" zu 6 Monaten Arrest verurtheilt wurde. Die Beobachtungen während dieser ganzen Zeit ergaben, dass S. im Verkehrston gesprochene

1) Lehrbuch 1887. S. 256 .

2) Observations cliniques: Revae mens. de Laryngologie 1886. S. 556.

3) Archiv. d'ophtalmol. 1885. p. 385.

4) A. f. A. u. O. Bd. IX. S. 337 .

4) 1. c. S. 32 . 
Worte manchmal ,ganz gut" hörte, manchmal wieder nur bei heftigem Anschreien oder gar nicht; ferner, dass er mit seiner Familie sprach und ,ganz gut" hörte. Eine Ueberraschung des Mannes im Schlafe war auch nicht möglich, da er sich immer wie leblos stellte und, ohne eine Miene zu verziehen, sich im Bette hin und her wälzen liess. Objectiv konnte ansser einer Einziebung des Trommelfelles nichts nachgewiesen werden. Die Hörprufungen blieben resultatlos, da er auf alle Fragen schwieg. Ein äratliches Gutachten spricht sich schliesslich dahin aus: "Man kann mit Gewissheit annehmen, dass die angebliche Taubheit des Mannes thatsăchlich nicht besteht, sondern fingirt ist."

Die sechsmonatliche Arreststrafe änderte in seinem Benehmen nichts; er blieb bei seiner Angabe, dass er seine Schwerhörigkeit nicht simulire. Die ununterbrochene, unauffällige Beobachtung konnte auch keine Resultate auf die Unrichtigkeit seiner Angaben ergeben. Es wurde neuerdings die Strafanzeige wider ihn veraulasst. Die in der neuerlichen Untersuchung eidlich einvernommenen Zeugen gaben übereinstimmend an, dass $\mathrm{S}$. seit seinem Wiedereinrüicken zur Escadron nach Abbüssung der sechsmonatlichen Arreststrafe auf eine Ansprache nur dann reagirte, wenn ihm ins rechte Ohr geschrien wurde. Auf Grund dieser und der in der 1. Untersuchung zu Tage getretenen Facten wurde er neuerdings zu 9 Monaten strengem GarnisonsArrest verurtheilt. Vor Bestätigung der Strafe wurde jedoch S. zufolge Verordnung des k. a. k. Militär-Obergerichtes zur "neuerlichen ärztlichen Bcobacbtung und Abgabe eines Gutachtens über die angebliche Schwerbörigkeit des Untersuchten im gegenwärtigen Zeitpunkte" dem spitale übergeben. Von diesem Spitale wurde dessen Abgabe in das hiesige Garnisons-Spital zur specialärztlichen Untersuchung beantragt und auch bewilligt. S. stand durch 5 Monate im hiesigen Spitale in Beobachtung. Der Befund war folgender:

Rechtes 0 hr: Gehörgang mittelweit, Trommelfell atrophisch, eingezogen, durchscheinend, Lichtkegel an der Basis verbreitert, unregelmässig; Randknickung; seichtes Grübchen in der Membrana fiaccida.

Linkes $0 \mathrm{hr}$ : Trommelfell durchscheinend, eingezogen, Lichtkegel nur an der Spitze.

Nase: Unterer Nasengang rechts weit, die Schleimhaut der unteren Muschel leicht geschwollen, mit eingetrocknetem, grünlichem Secrete bedeckt. Am Rachendach flache Granula mit schleimigem Eiter bedeckt.

Bei den oftmals vorgenommenen Hörprüfungen war das Resultat sowohl in Bezug auf Flüster- wie laute Stimme beiderseits immer ein vollkommen negatives. Sehr laute Stimme wurde am rechten Ohre noch percipirt, jedoch nur aus unmittelbarer Nähe. Die Uhr wurde beiderseits weder in Luft-, noch Knochenleitung gehört. Prüfung mittelst der ganzen Reibe der Stimmgabeltöne, auch Harmonika, ergaben beiderseits ein negatives Resultat. Alle auf Hörprüfungen bezüglichen Fragen wurden dahin beantwortet: ,Am linken Ohre höre ich gar nichts, am rechten ein Bissel", oder ,ich höre nichts". An die Tafel geschriebene Fragen beantwortet derselbe uur ungern und mit etwas stotternder Sprache, oder er hüllt sich in beharrliches Schweigen. Die fortgesetzte unauffällige Beobachtung wăhrend der ganzen Zeit sowohl von Seiten der Aerzte und des Wartepersonales als auch von Seiten seiner Zimmergenossen ergaben kein anderes Resultat, als dass S. nur dann auf Fragen reagirte, wenn ihm diese mit schreiender Stimme in das rechte Ohr gesprochen wurden. Auch Versuche, ihn des Nachts mittelst Anfangs leiser und immer lauterer Stimme aus dem Schlafe zu wecken, blieben erfolglos. Er reagirte auch nicht darauf, dass seinem Wärter vor ihm gesagt warde, ,S. werde morgen operirt nnd darf daber kein Frubstuck bekommen." Wiederholte Ermahnungen und auch angedrohte Strafen änderten an diesem seinem Verhalten während der ganzen Beobachtungsdauer nichts. Fieberbewegungen, Kopfschmerz, Schwindel, schwankender Gang oder Erbrechen wnrden nie beobachtet und wurden auch nicht unangenehme subjective Gehörempfindungen geklagt. Die Untersuchung des Gesichtsfeldes ergab eine concentrische Einengung desselben; ob sonst eine Störung der Sensibilität vorhanden war, war nicht zu eruiren.

In Bezug auf die Verordnung: Abgabe eines Gutachtens über die an- 
gebliche Schwerbörigkeit im gegenwärtigen Zeitpunkte wurde nun folgendes Gutachten abgegeben:

Die von dem Untersuchten wahrgenommenen Erscheinungen sind in 2 Reihen zu theilen:

A) in die 1. Reihe, enthaltend jene Symptome, welche durch das Auge des Untersuchenden allein, also unabhängig ron der Mitwirkung des Untersuchten, constatirt wurden und

B) in die 2. Reihe, enthaltend jene Symptome, welche nur unter Mitwirkung des Untersuchten zu erbeben waren (Angaben über die Gehörempfindung bei den Hörprüfungen).

ad A) Aus dem Befunde geht hervor, dass

a) ein chronischer Nasenrachenkatarrh besteht. Dieses Leiden besteht seit Jahren, aller Wahrscheinlichkeit nach seit Kindheit (Granula am Rachendach). Durch Fortleitung des Katarrbs durch die Tuben ist es

b) zu einem beiderseitigen chronischen Mittelohrkatarrh gekommen (Atrophie und Einziehung beider Trommelfelle).

Auch dieser Mittelohrkatarrh besteht jedenfalls seit längerer Zeit, hat aller Wahrscheinlichkeit nach schon vor der im December 1894 erlittenen Ohrfeige bestanden, und waren die dadurch hervorgerufenen Verănderungen des Trommelfelles (leichtere Zerreissbarkeit infolge Schwund) geeignet, auch infolge einer minder wachtigen Ohrfeige schon einen Trommelfellriss zu ermöglichen.

Die sub $a$ und $b$ argegebenen Krankheitszustände sind jedoch nur in einem solchen Grade vorhanden, dass durch dieselben allein erst eine Herabsetzung des Hörvermögens beider Ohren in geringem Grade bewirkt werden kann und aller Wahrscheinlichkeit nach bewirkt wird, eine Taubheit des linken und eine hochgradige Schwerhörigkeit des rechten Ohres jedoch sind durch diese objectiv constatirten krankhaften Verănderungen nicht zu erklären.

ad B) Hier kommt zunächst die Frage in Betracht: Können die bei den Hörprüfungen gemachten Angaben des Untersuchten auf Wahrheit beruhen?

Seit der Abbüssung seiner sechsmonatlichen Arreststrafe konnte der Untersuchte weder während der Dienstleistung bei der Escadron, noch während seiner Beobachtung in den Spitälern, im Ganzen während eines Zeitraumes von 10 Monaten, jemals ertappt werden, dass er besser gehört hătte, als er selbst bei den Hörpräfungen und sonst zugab.

Von wissenschaftlichem Standpunkte ans kann die Unrichtigkeit seiner Angaben nicht bewiesen werden; denn wenn auch die Taubheit des linken und hochgradige Schwerhörigkeit des rechten Ohres durch den Spiegelbefund nicht $\mathrm{za}$ erklären ist, kann dieselbe auf eine tiefer liegende Affection zurückgeführt werden.

Labyrinthaffectionen kommen hierbei wohl nicht in Betracht, da die sie begleitenden Nebenerscheinungen: Kopfschmerz, Schwindel, taumelnder Gang, Uebelkeit, Erbrechen, subjective unangenehme Gebörsempfindungen an dem Untersuchten weder vor seiner Abgabe hierher noch hierorts beobachtet wurden.

Dagegen sind die tiefer liegenden krankhaften Affectionen des Gehörnerven, bezw. des im Gehirne liegenden Centralorganes für das Hörvermögen - insbesondere eine Neurose des Gehirnnerven - geeignet, die Resultate der Hörprüfungen wissenschaftlich zu erklären. Das Hörvermögen ist dabei nicht selten graduellen Schwankungen unterworfen, und es sind auch zeitliche und dauernde Heilungen solcher Neurosen beobachtet worden. Zumeist pflegen solche Neurosen bei Personen aufzutreten, welche auch anderweitige functionelle Nervenstörungen - hysterische Erscheinungen - zeigen. Zu bemerken ist, dass bei derartig belasteten Individuen oft selbst geringgradige Verănderungen des äusseren und mittleren Ohres zu solchen schweren und hartnäckigen Neurosen Anlass geben. Es kann demnach vom wissenscbaftlichen Standunkte die Gesammtheit der beobachteten abnormen Erscheinungen als chronischer Nasen-, Rachen-, Tuben- und Mittelohrkatarrh beiderseits, dannals Hörnervenneurosemit 
Taubheit links und Herabsetzung derHörweitefür lante Sprache auf unmittelbare Nabe rechts bezeichnet werden.

Die weitere in Betracht kommende Frage lantet: Ist Simulation bewiesen oder ausgeschlossen?

Nach den in der Anamnese angeführter Daten wurde S. in der Zeit von Anfang Januar 1895 bis ungefäbr Ende April 1895 wiederbolt der Simulation überwiesen.

Die Untersuchung hat keine objectiven - obne Hinznthun S.'s entstandenen - Beobachtungen ergeben, welche die Taubheit der linken und hochgradige Schwerhörigkeit des rechten Obres nothwendig folgern lassen würden.

$\mathrm{S}$. hat sich wiederholt bei den vorgenommenen Untersuchungen hier und auch im früheren Spitale hochgradïg widerwillig, stützig und widerspenstig gezeigf, und seine Art, immer dieselben Antworten zu geben oder Antworten überhaupt zu verweigern, muss sehr den Verdacht wecken, dass er zielbewusst die Gelegenheiten meide, sich anf einem Widerspruche ertappen zu lassen.

So schwer jedoch diese Verdachtsgründe wiegen mögen, um subjective Meinungen über Simnlation zu begründen, sind sie doch nicht geeignet, die Simulation als wirklich vorhanden zu beweisen. Ferner muss der Mann, weil er vor ca. 11/2 Jabren simulirte, nicht auch dermalen simuliren; seit 31. April 1895 ist er ja trotz vielfältiger langer Beobachtung einer Simulation vicht mehr überfübrt worden. Es wurde auch schon angeführt, dass sich seine Angaben aber das Hörvermögen mit den wissenschaftlichen Erfahrungen nicht in Widerspruch setzen, dass selbst seine frühere Simulation mit einem wissenschaftlich als Acusticusneurose zu bezeichnenden Krankheitszustande in plausible Verbindung gebracht werden kann ${ }^{1}$ ).

Organische Veränderungen am Gehörorgane waren also hier zu Anfang schon nachzuweisen, functionelle Störungen als Folge des Traumas sind unstreitig ebenfalis hier. Nun entsteht die Frage: Hat sich das Bild der traumatischen Neurose anf dem Boden der materiellen Veränderung entwickelt oder ist es rein?

Ich neige in dem eben beschriebenen Falle zur letzteren Anschauung. Es wurden niemals Gleichgewichtsstörungen, Sehwindel, Erbrechen oder subjective Gehörsempfindungen, also Veränderungen, welche auf eine organische Veränderung des Labyrinthes schliessen liessen, geklagt. Die Taubheit, resp. Schwerhörigkeit trat nicht sofort nach der Verletzung auf, sondern erst nach einiger Zeit (psychische Incubation). Die Annahme von Möbius, welcher sich auch Gradenigo anschliesst, dass bei der Hysterie Schwindelanfälle fehlen, als einziges Kriterium für die differentielle Diagnose zwischen materieller Veränderung und functioneller Störung aufzustellen, ist sicherlich gewagt. Wenn dies auch fiir ein einzelnes Symptom, wie Schwindel, Gleichgewichtsstörungen ${ }^{2}$ ) zngegeben werden muss, so kann doch

1) Vorgeschichte und Begutachtung wurden ausführlicher mitgetheilt, um auch fremder Beurtheilung zugänglich zu sein.

2) Kretschmann hält dies ausschlaggebend für eine Labyrinthblutung. Dieses Archiv. Bd. XXIII. 
266 XXII. BIEHL, Die Beurtheilung ein- und beiderseitiger Taubheit.

ein Gleiches sicherlich nicht bebauptet werden, wenn ein ganzer Symptomencomplex nachzuweisen ist.

Dass sich durch organische Labyrinthläsion allmählich ein Allgemeinzustand ausbilden kann, der vollständig dem Bilde der traumatischen Hysterie entspricht, beweist Brieger durch ein Beispiel (1. c.):

Nach einer Kopfverletzung tritt Bewusstlosigkeit and rechtsseitige Ohrblutung ein. Nach Wiederkehr des Bewusstseins doppelseitige Schwerhörigkeit, beiderseits aufgehobene Knochenleitung; rechtsseitige traumatische Trommelfellraptur. $\mathrm{Es}$ bestand rechts bleibende hochgradige Schwerhörigkeit, ausserdem häufige Schwindelanfälle; links trat nach etwa 14 Tagen erhebliche Besserung ein. Man darf annehmen, dass das Trauma links eine Commotion, rechts eine ausgedehntere Hämorrhagie zur Folge gehabt hat. Nach einem halben Jahre findet sich derselbe locale Befund; daneben tiefe gemüthliche Depression, hypochondrische Vorstellungen, objectiv beiderseits concentrische Gesichtsfeldeinengung - kurz, das typische Bild der traumatischen Neurose. 